\title{
MODIFICATION OF DECODER FOR 2-D WAVELENGTH/TIME OPTICAL CDMA SYSTEM BY OPTICAL HARD-LIMITERS
}

\author{
Jozef Chovan ${ }^{1,2}$, František Uherek ${ }^{1,2}$ \\ 'International Laser Center, Ilkovičova 3, 81219 Bratislava, Slovak Republic, \\ e-mail:chovan@ilc.sk \\ ${ }^{2}$ Slovak University of Technology, Faculty of Electrical Engineering and Information \\ Technology, Department of Microelectronics, Ilkovičova 3, 81219 Bratislava, Slovak \\ Republic,e-mail: uherek@elf.stuba.sk
}

\begin{abstract}
We present the modification of decoder for 2-D Wavelength/Time (W/T) Optical Code Division Multiple Access (O-CDMA) system by optical hardlimiters. The performance improvement of the system using proposed decoder modification is shown by numerical simulation of the average Signal to interference difference and Bit error probability as a function of active user number and weight of codeword for the different topology of the decoder. The influence of non-ideal properties of the used optical hardlimiters upon the bit error probability in system is studied.
\end{abstract}

\section{INTRODUCTION}

Optical Code Division Multiple Access (O-CDMA) system is advance alloptical multi-channel scheme for fiber optic networks, which allows asynchronous transmission mode and accession to the optical network simultaneously. One of the most important problems for O-CDMA systems is the Multiple-access interference (MAI), which limits the number of active users in the system. We proposed the modification of 2-D W/T decoder [1] by placing the second optical hard-limiter (O-HL) after its last optical coupler (O-HL2 in Fig.1). The $\mathrm{O}-\mathrm{HL}$ is a nonlinear all-optical threshold device. It has two valued output optical intensity, which depends on the optical intensity at the input [2]. The topologies 


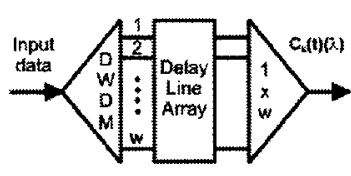

ENCODER

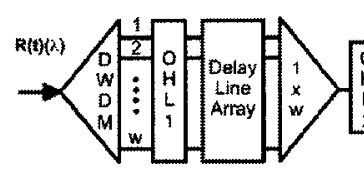

DECODER

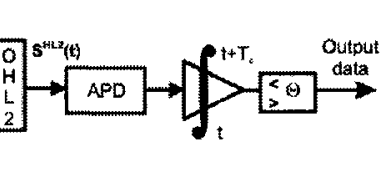

RECENER

Figure 1. 2-D W/T O-CDMA encoder and modified decoder with O-HLs

of 2-D W/T encoder and modified 2-D W/T decoder are shown in Fig.1. The first $\mathrm{O}-\mathrm{HL}$ (O-HL1) is placed into the each delay lines of delay line array of the decoder, which clips back the amplitudes of the unwanted created optical pulses at the same wavelength. The second O-HL (O-HL2) selects the autocorrelation pulse from the output optical pulses sequence, which determines the transmitted information data bits.

In the performance evaluation of the 2-D W/T O-CDMA system we assume an implemented passive O-HLs proposed in [2] by L. Brzozowski and E. H Sargent. The proposed passive O-HL is a periodic structure consisting of alternating layers of materials possessing different optical Kerr nonlinearity [2]. We used the approximation their properties by two ideality parameters IP1 and IP2 made in [3]. These two parameters characterize the measure of O-HL non-ideal properties. An intensity modulation and direct detection by APD optical receiver are assumed. Bit error probability (BEP) is used for performance evaluation of the system. BEP is calculated by noise model of APD optical receiver published in [4]. This model contains the excess noise of APD, thermal noise and MAI noise. In this paper we have used the average signal-to-interference difference (SID) as the MAI merits calculated by the following proposed numerical model of the 2-D W/T O-CDMA system.

\section{SYSTEM MODEL}

We consider an incoherent optical O-CDMA system with 2-D W/T codewords. $N$ users share the same optical medium usually, but not exclusively, in a star topology. Each $k$-th user's information bit form is encoded into a 2-D W/T codeword

$$
C_{k}(t)(\lambda)=\sum_{i=1}^{m} \sum_{j=1}^{n} c_{k}[j][i] p_{j}\left(t-j T_{c}\right) v_{i}\left(\lambda-i \lambda_{c}\right),
$$

where $n$ is the length of the codeword in the time domain, $T=n T_{c}$ is the time duration of the information data bit, $m$ is number of the available wavelength, $\Delta \lambda=$ $m \lambda_{c}$ is the spectral width of optical information data bit and $C_{k}=[j][i] \epsilon\{0,1\}$, for $l \leq j<n, l \leq i<m$, is the $j, i$-th chip pulse of the $k$-th user's codeword. Let 
$C_{k}=\left\{c_{k}[1][1], c_{k}[1][1], \ldots, c_{k}[m][n]\right\}$ be a matrices representing the discrete form of the 2-D W/T codeword. Further, the chip signaling waveform $p_{j}(t)$ is assumed to be a unit-amplitude rectangular pulse of the chip time duration $T_{c}$ and the spectral waveform $v_{i}(\lambda)$ is assumed to be a unit-amplitude rectangular pulse of the spectral width $\lambda_{c}$. Each user transmitter broadcasts its encoded signal to all receivers in the system by star topology of the system. The received signal is a sum of all active $N$ users' transmitted signals

$$
R(t)(\lambda)=\sum_{k=1}^{N} b_{k} C_{k}\left(t-\tau_{k}\right)(\lambda),
$$

where $b_{k} \in\{0,1\}$ is the $k$-th user's information bit and $0 \leq \tau<T$ is the time delay for $k=1, \ldots, N$. The decoder performs the correlation function of the sequence of incoming optical chip pulses. The output signal of desired $k$-user's optical decoder is thus

$$
S_{k}(t)=C_{k}(t)(\lambda) R(t)(\lambda)
$$

If the second O-HL is applied to the output signal of optical decoder, then the output signal of the optical decoder can be express as

$$
S_{k}^{H L 2}(t)=I_{H L 2}\left(S_{k}(t)\right)
$$

where $I_{H L 2}$ is transmission function of O-HL [3]. The output signal of $k$-th user decoder is sequence of optical chip pulses with different amplitudes. It can be expressed as

$$
S_{k}(t)=\sum_{i=1}^{2 n} a_{k}^{l}[i] p_{i}\left(t-i T_{c}\right),
$$

where $a_{k}^{l}$ is a normalized amplitude of the optical chip pulse of $\mathrm{i}$-chip time, $l \epsilon\{0,1\}$ for $b_{k}=1$ and $b_{k}=0$ transmitted data bit, respectively. Let $A_{k}^{l}=\left\{a_{k}^{l}[1], a_{k}^{l}\right.$ $\left.[2], \ldots, a_{k}^{l}[n]\right\}$ be a vector representing the discrete form of the output chip pulse sequence of $k$-th user decoder. Let

$$
\begin{aligned}
& a_{k, \max }^{0}=\max \left\{a_{k}^{0}[1], a_{k}^{0}[2], \ldots \ldots \ldots \ldots \ldots a_{k}^{0}[n]\right\}, \\
& a_{k, \max }^{1}=\max \left\{a_{k}^{1}[1], a_{k}^{1}[2], \ldots \ldots \ldots \ldots \ldots a_{k}^{1}[n]\right\} .
\end{aligned}
$$

In this paper the average SID is defined as

$$
S I D=\frac{1}{N} \sum_{k=1}^{N} a_{k, \max }^{1}-a_{k, \max }^{0} .
$$

The probability that a specified number of photons are absorbed from an incident optical field by an optical detector over a chip interval $T_{c}$ is given by a Poisson distribution [4]. The photon absorption rate $\lambda_{s}$ is given by 


$$
\lambda_{s}=\frac{\eta P_{r} \lambda}{h c}
$$

where $\eta$ is the APD quantum efficiency, $P_{r}$ is received laser power, $\lambda$ is central wavelength and $h$ is Plank's constant. Hence, due to "one" and "zero" data bit transmission, the mean $\mu_{b(1,0)}$ and variance $\sigma_{b(1,0)}$ of number of received photons [4] can be written as

$$
\begin{aligned}
& \mu_{b(1)}=M_{A P D} T_{C}\left[w \lambda_{s}+\left(\lambda_{0}+\frac{I_{b}}{e}\right)\right]+T_{C} \frac{I_{s}}{e}, \\
& \sigma_{b(1)}^{2}=M_{A P D}^{2} F_{e} T_{C}\left[w \lambda_{s}+\left(\lambda_{0}+\frac{I_{b}}{e}\right)\right]+\left(T_{C} \frac{I_{s}}{e}+\sigma_{t h}^{2}\right), \\
& \mu_{b(0)}=M_{A P D} T_{C}\left[(w-S I D) \lambda_{s}+\left(\lambda_{0}+\frac{I_{b}}{e}\right)\right]+T_{C} \frac{I_{s}}{e}, \\
& \sigma_{b(0)}^{2}=M_{A P D}^{2} F_{e} T_{C}\left[(w-S I D) \lambda_{s}+\left(\lambda_{0}+\frac{I_{b}}{e}\right)\right]+\left(T_{C} \frac{I_{s}}{e}+\sigma_{t h}^{2}\right),
\end{aligned}
$$

where $T_{C}$ is a time chip interval, $n$ is a length of codewords and $\lambda_{0}$ is photon absorption rate due to a background light. The values of the parameters of APD detector is used the same as in [4]. In this performance evaluation we assume Gaussian approximation at the APD output. An optimum receiver uses the value of threshold [4], which minimizes the overall error probability. The average $B E P$ is given by

$$
B E P=\frac{1}{2}\left[\operatorname{erfc}\left(\frac{\mu_{b(1)}-\theta_{o p t}}{\sigma_{b(1)}}\right)+e r f c\left(\frac{\theta_{o p t}-\mu_{b(0)}}{\sigma_{b(0)}}\right)\right] .
$$

\section{NUMERICAL RESULTS}

The obtained numerical result of BEP variance as a function of number active users and weight of codewords for different values of IP2 and topology of decoder is calculated by PC software. PC software calculates the average value of BEP by equations (1-14). At the beginning of the BEP calculation, 2-D generalized multi-wavelength prime codewords (GMWPC) are generated for each user of the 2-D W/T O-CDMA system by algorithm proposed in [5]. The independent input parameters of this algorithm are weight of codewords $w$ and a set of prime $p_{k} \geq p_{k-l}$ $\geq p_{k-2 \ldots} \geq p_{l} \geq w$. The output of the algorithm is $p_{I} p_{2 \ldots} p_{k}$ GMWPC with these parameters $\left(w \times p_{1} p_{2} p_{k}, w, 0,1\right)$, zero autocorrelation side lobes, cross-correlation functions of at most 1 . The choosing of the active users is random generated 
up to required number. The time delay between each user's codewords, $\tau_{c}$ in equation (2), is random generated by Gaussian distribution. After that it calculates the output optical pulse sequence of each active user 2-D W/T O-CDMA decoder for "one" and "zero" data bit transmission and the average value of SIR and BEP in 2-D W/T O-CDMA system with specified parameters.

The two periods of input and three periods of output signals of the 101 -th user decoder in the O-CDMA system with 150 active users and $(7 \times 343,7,0,1)$ GMWPC are shown in Figs. 2 - 5. Figure 2 shows the input and output signal of this decoder without any O-HLs for "one" data bit transmission in time domain. The input signal is a sum all active $N$ users' transmitted codewords (Eq. 2) and it contain optical chip pulses with normalized amplitudes more grater than one. It can be seen that, when O-CDMA decoder is without any O-HL, the amplitude of the created autocorrelation optical chip pulse does not have the defined normalized amplitude by used GMWPC and some other optical chip pulses have the same amplitude as the autocorrelation optical chip pulse. Thus in this case it is not possible to detect transmitted data bit by threshold device at the end of optical receiver, which is determined by autocorrelation pulse.
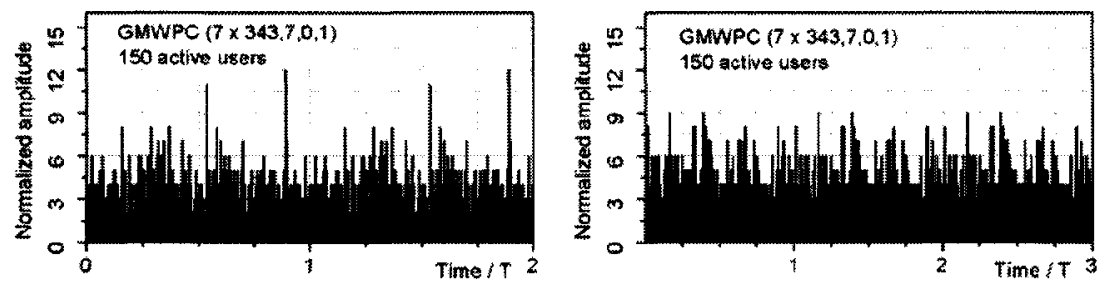

Figure 2. Input and output signals of the decoder without any O-HL

The left part of Fig. 3 shows the first delay line signal of decoder, which contains the unwanted optical pulses created by MAI at the same wavelength with normalized amplitude greater than 1 . Therefore the first O-HL1 is placed into the each delay lines, which clips back the normalized amplitudes of the unwanted created optical pulses to 1 (the right part of Fig.3).

Output signals of the decoder with only first O-HL1s for "one" data bit transmission are shown in the left part of Fig.4. In this case the amplitude differences between autocorrelation and the other optical pulses are not significant. In the case, when decoder is only with the second O-HL2, the decoder output signal contains the unwanted optical pulse with the same normalized amplitude than autocorrelation pulses (right part of Fig. 4).

Output signals of the decoder with both O-HLs for "one" and "zero" data bit transmission are shown in Fig. 5. In this case of the decoder topology it is possible to detect the transmitted information data bit by threshold device of optical receiver. 

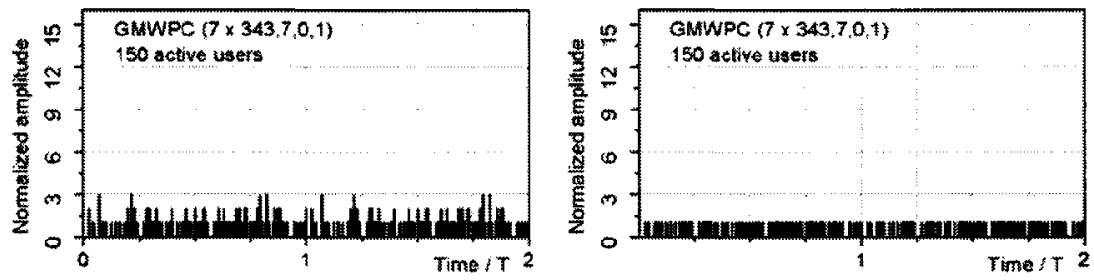

Figure 3. Signal of the decoder at first wavelength without any and with first O-HL1
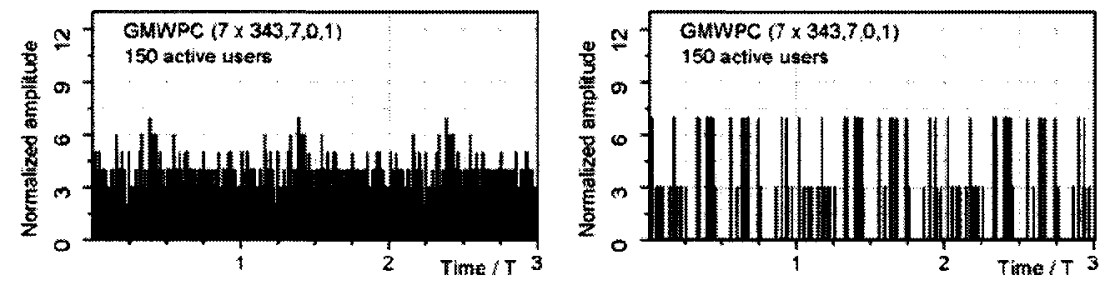

Figure 4. Output signals of the decoder with only first O-HL1 and with only second O-HL2
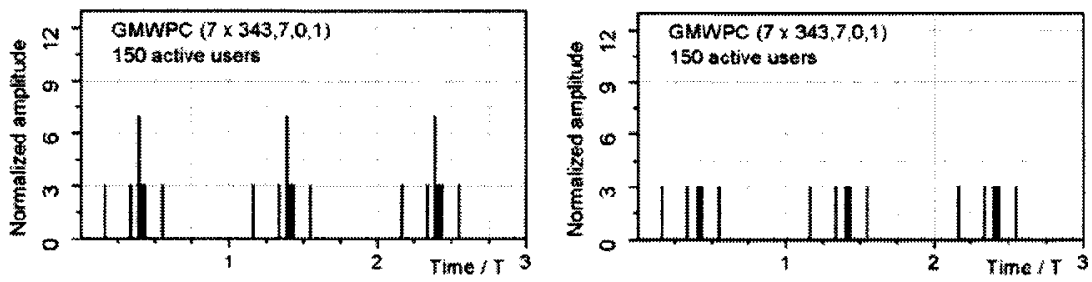

Figure 5. Output signals of the decoder with both O-HLs for "one" and "zero" data bit transmission

The obtained numerical result of average value of SID (Eq. 8) variance as a function of number active users and weight of codewords for different values of IP2 and the topology of the decoder can be seen in Fig. 6. The improvement of SID value by using decoder with both O-HL can be seen.

Figure 7 shows the dependence BEP versus number of the active users in the O-CDMA system with the decoder with only first O-HL (HL1) and with both O-HLs (HL1_HL2) for the fixed weight of codewords $w=9$ and fixed photon absorption rate $\lambda_{s}=1 E 14$. This dependence is calculated for three different value of IP2 $(0.25,0.5$, and 0.75$)$. Figure 7 depicts the improvement of BEP by using decoder with both O-HLs. The value of BEP for number of active users $N=200$ and $I P 2=0.75$ is changed from $10 \mathrm{E}-1$ to $10 \mathrm{E}-8$ by using the second $\mathrm{O}-\mathrm{HL}$. Figure 7 also shows the influence of non-ideal properties of the used O-HL to the BEP. The influence of the second $\mathrm{O}-\mathrm{HL}$ to the $\mathrm{BEP}$ is greater when its properties convergent to ideal. 

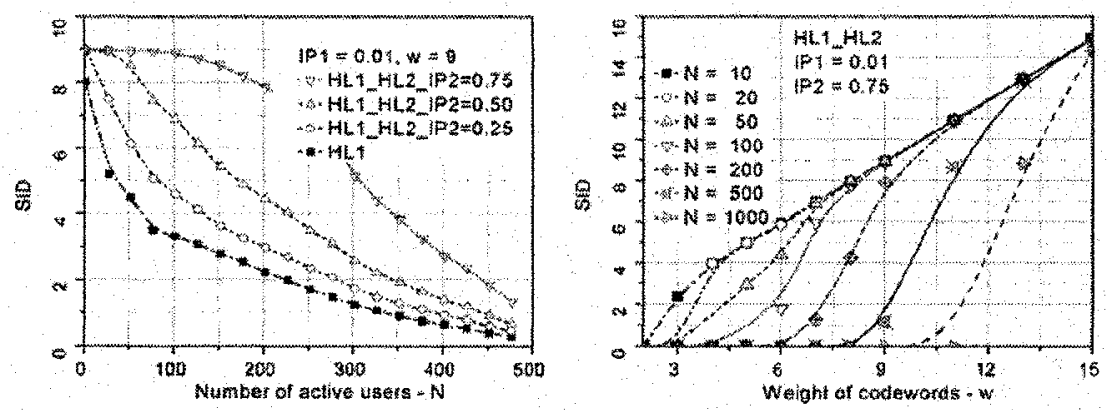

Figure 6. SID versus $\mathrm{N}$ and $\mathrm{w}$ for various values of IP2 and the topology of the decoder

Figure 8 shows the variation of BEP as a function of the weight of the codeword for four different number of active user (50,100, 200, and 500). It assumes decoder with both O-HLs and $\lambda_{s}=2 E 13$. From the dependence, for the case $N=50$, it can be seen that the dominant effect to $B E P$ has the MAI for the weight of the codeword approximately up to 6 . The increasing of value of BEP, for the weight of codeword greater than 6 , is caused by decreasing of the mean signal photon count per $T_{c}$, because the length of the codeword is increasing and thus $T_{c}$ is decreasing. From this dependence it can be seen that the optimum weight of the codewords exits for achieving the minimum value of the $B E P$ for specified parameters of the O-CDMA system.

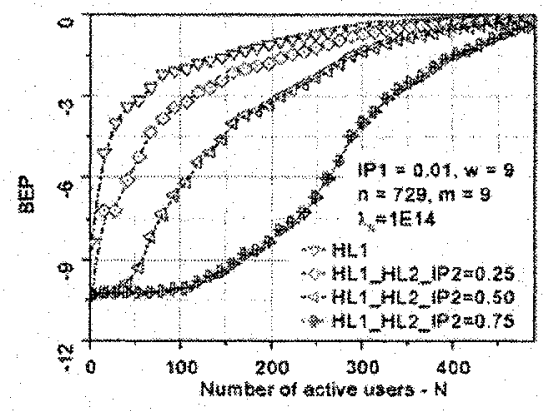

Figure 7. BEP versus $\mathrm{N}$ for the decoder with first one and both O-HLs and for various value of IP2

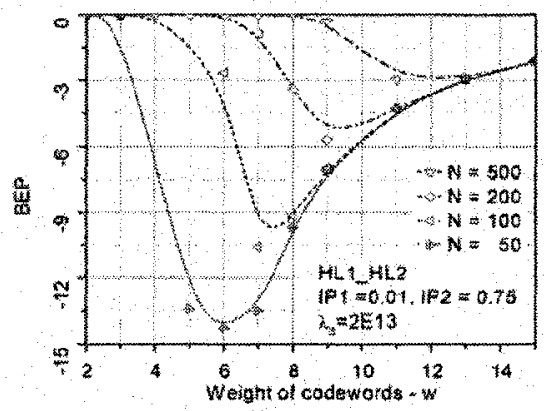

Figure 8. BEP versus w with decoder with both O-HLs for different number of active users $\mathrm{N}$ 


\section{CONCLUSIONS}

The performance improvement of 2-D W/T O-CDMA system by using decoder with two O-HLs and the influence of O-HL properties have been shown by numerical modeling of the average Signal to interference difference and Bit error probability as a function of active users number and weight of codewords for different decoder topology.

The performance evaluation has shown that the dependence of bit error probability versus the weight of codewords has a local minimum, what allows choosing an optimum weight of the codewords for achieving the minimum bit error probability in 2-D W/T O-CDMA system for given system parameters.

\section{ACKNOWLEDGMENTS}

The work at the International Laser Center was supported by the project No.1/0130/03 and at the Department of Microelectronics of Faculty of Electrical Engineering and Information Technology by the project No.1/0152/03 both from the Slovak Ministry of Education.

\section{REFERENCES}

[1] J. Mendez, et al., "Design and Performance Analysis of Wavelength/Time (W/T) Matrix Codes for Optical CDMA," J. Lightwave Technol., vol. 21, pp. 2524-2533, Nov. 2003.

[2] L. Brzozowski, E. H. Sargent, "Nonlinear distributed-feedback structures as passive optical limiters," J. Opt. Soc. Am. B., vol.17, pp.1360-1368, 2000.

[3] J. Chovan, F. Uherek, P. Hábovčík, "Performance analysis of an optical CDMA system with non-ideal optical hard-limiters," Optics Communication, vol. 216, pp. 289-297, 2003.

[4] A.Srivastava, et al., "Performance evaluation of PIN+OA and APD receiver in multiwavelength CDMA and WaCDMA networks," Optics Communication, vol. 191, pp. 55-66, May 2001.

[5] G.C. Yang, W.C.Kwong, "Performance Comparison of Multiwavelength CDMA and WDMA - CDMA for Fiber-Optic Networks," IEEE Trans. Communication, vol. 45, pp. 1426-1434, Nov. 1997. 